A. RAS-SABIDÓ

KODAI MATH. J.

14 (1991), 477-484

\title{
KILLING FIELDS PRESERVING TOTALLY GEODESIC, CODIMENSION-ONE FOLIATIONS
}

\author{
ANTONI RAS-SABIDÓ
}

\section{$\S 1$. Introduction}

Let $M$ be a complete manifold, endowed with a codimension-one foliation $\mathscr{T}$. We want to study the Lie algebra $\mathcal{G}$ of Killing fields preserving the foliation (i.e., Killing fields such that the isometries of their one-parameter group send leaves of $\mathscr{F}$ onto leaves of $\mathscr{I}$ ).

In [5], Johnson and Whitt proved that when the foliation is totally geodesic (i.e., leaves are totally geodesic submanifolds) and all the leaves are compact, then any Killing field preserves $\mathscr{F}$. Later, Oshikiri (see [7]) proved the same result for the case when the manifold is compact and $\mathscr{T}$ is totally geodesic. Nevertheless, in the general case all Killing fields do not preserve foliations. For example, in the euclidean plane foliated by lines parallel to the $0 \mathrm{X}$-axis, Killing fields associated to rotations do not preserve the foliation.

This paper is part of author's doctoral thesis. He wishes to thank his advisor Professor C. Currás-Bosch for his aid and constant support.

\section{§2. Totally geodesic foliations}

First of all, let us recall that any codimension-one foliation which admits an orthogonal Killing field must be totally geodesic (see [3] for instance). For this reason, from now on we shall only consider totally geodesic foliations. The universal cover of a manifold with such a structure verifies the following

THEOREM 1. (see [2]) Let $(M, \mathscr{T})$ be a complete manifold with a codimensionone, totally geodesic foliation. Let $\tilde{M}$ be the universal cover of $M$. Then $\tilde{M}$ is trivially foliated as $\hat{L} \times \boldsymbol{R}$, where $\widetilde{L}$ is the universal cover of any leaf and the induced metric reads $d s_{\tilde{M}}^{2}=d s_{\tilde{L}}^{2}+f^{2} d \tilde{t}^{2}$, where $f: \tilde{M} \rightarrow(0, \infty)$ is a $C^{\infty}$ function.

In order to simplify calculations, it will be convenient to give a characterization of Killing fields preserving foliations. Let $(M, \mathscr{T})$ be a complete manifold with a codimension-one, totally geodesic foliation. With the notations of Theorem 1,

Partially supported by the CAYCIT, ref. 1085-84

Received January 16, 1991; revised May 28, 1991. 
Proposition 1. Any Killing field $X \in i(\tilde{M})$ is of the form $X=X^{t}+\lambda \partial t$, where

i) $X^{t}$ is a Killing field on $\widehat{L}$ with respect to $d s_{\tilde{L}}^{2}$;

ii) $X^{t} f=-\partial_{t}(\lambda f)$;

iii) $f^{2} \cdot(Y \lambda)=\left\langle Y,\left[X^{t}, \partial t\right]\right\rangle, \forall Y \in T(\mathscr{I})$ with $[Y, \partial t]=0$.

Moreover, $X$ preserves the foliation if and only if it verifies also that

iv) $Y \lambda=0, \forall Y \in T(\mathscr{F})$, or, equivalently, $\left[X^{t}, \partial t\right]=0$.

Proof. See Propositions 1.1 and 1.2 of [4].

Passing, if necessary, to a 2-fold cover, we may suppose foliations to be transversally oriented. Thus, from now on, we will assume this fact and call $N$ the normal field to $\mathscr{F}$ (i.e. an unitary vector field orthogonal to $\mathscr{F}$ ). According to the characteristics of the function $f$ in Theorem 1 , we will consider two cases:

i) $Y f=0, \forall Y \in T(\mathscr{I})$. Then, $\mathscr{F}$ is a bundle-like foliation.

ii) Otherwise, we have the general case.

There is not much to say about bundle-like, totally geodesic foliations. Thus, we begin with case ii): let us assume for the moment that $f$ is not constant in the leaves. Our goal now is to give the best bound possible for the dimension of $\mathcal{G}$, the Lie algebra of Killing fields preserving the foliation. First of all, let us give an upper bound for the dimension of its subalgebra $\mathcal{G}^{t}$ of Killing fields tangent to $\mathscr{T}$. If $n=$ dimension $\mathscr{T}$, it is clear that dimension $\mathcal{G}^{t} \leqq(1 / 2) n$ $(n+1)$. We want to show that actually dimension $\mathcal{G}^{t} \leqq(1 / 2) n(n-1)$. Before this, we need some Lemmas.

LEMMA 1. Let $M$ be a complete n-dimensional manifold, endowed with a foliation $\mathscr{I}$ of dimension $m<n$ and let $X \in i(M) \cap T(\mathscr{I})$. If there is some leaf $L$ in which $X_{1 L} \equiv 0$, then $X \equiv 0$.

Proof. Let $\left(\phi_{t}\right)$ be the one-parameter group associated to $X$. We shall see that, for any $p \in L,\left(\phi_{t}\right)_{* p}=I d, \forall t$. In a neighborhood of $p$, let $\left(\partial x^{1}, \cdots, \partial x^{m}\right.$, $\left.\partial y^{1}, \cdots, \partial y^{n-m}\right)$ be a basis such that the leaves of $\mathscr{I}$ are locally of the form $\left\{y^{1}=c t t ., \cdots, y^{n-m}=c t t\right.$. $\}$. As in [9], we can modify it to a new basis $\left(\partial x^{1}, \cdots\right.$, $\left.\partial x^{m}, \nu^{1}, \cdots, \nu^{n-m}\right)$, with $\nu^{\jmath}=y \partial^{j}+\sum b_{j i} \partial x^{i}, \forall j$ and such that $\left\langle\nu^{\jmath}, \partial x^{2}\right\rangle=0, \forall i, j$. Now :

$$
\begin{aligned}
& \left(\phi_{t}\right)_{* p}\left(\partial x^{i}\right)=\partial x^{i}, \quad\left(\phi_{t}\right)_{* p}\left(\partial y^{j}\right)=\partial y^{j}+\sum_{i=1}^{m} \mu_{\imath j} \partial x^{i}, \\
& \left(\phi_{t}\right)_{* p}\left(\nu^{\jmath}-\partial y^{j}\right)=\nu^{\jmath}-\partial y^{\jmath} \quad\left(\operatorname{as}\left(\nu^{\jmath}-\partial y^{\jmath}\right) \in T(\mathscr{F})\right),
\end{aligned}
$$

because $X$ is tangent to $\mathscr{I}$ and vanishes at the leaf $L$. Thus,

$$
\left(\phi_{t}\right)_{* p}\left(\nu^{\jmath}\right)=\nu^{\jmath}+\sum_{i=1}^{m} \mu_{\imath j} \partial x^{\imath} .
$$

$\left(\phi_{t}\right)_{*}$ is an isometry and preserves the foliation. Then $\left(\phi_{t}\right)_{* p}\left(\nu^{\jmath}\right)$ must be or- 
thogonal to $\mathscr{F}$. It follows from (1) and the expression of the riemannian metric in the basis $\left(\partial x^{1}, \cdots, \partial x^{m}, \nu^{1}, \cdots, \nu^{n-m}\right)$ that $\left(\phi_{t}\right)_{* p}\left(\nu^{j}\right)=\left(\nu^{j}\right), \forall j$. That is, $\left(\phi_{t}\right)_{* p}$ $=I d_{T_{p} M} . \phi$ was an arbitrary point and the manifold is complete, thus $\left(\phi_{t}\right)=I d$,

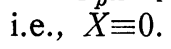

LEMMA 2. Let $M$ be a complete manifold and $\mathscr{I}$ a subalgebra of $i(M)$. Assume that $\forall p \in M$, dimension $\mathscr{T}_{p} \leqq m$. Then dimension $\mathfrak{I} \leqq r=:(1 / 2) m(m+1)$.

Proof. Let $p \in M$ with dimension $\mathscr{I}_{p}=m$. We can choose $m$ fields, $X_{1}, \cdots$, $X_{m} \in \mathscr{I}$, independent (as vectors) in a neighborhood $U$ of $p$. In $U$ let $\mathcal{S}$ be the distribution generated by $\left\{X_{1}, \cdots, X_{m}\right\}$. It is easy to see that $\mathcal{S}$ is involutive and then defines a foliation $\mathscr{F}_{U}$ of dimension $m$ in $U$. If dimension $\mathscr{T}>r$, let $Y_{1}, \cdots, Y_{r+1} \in \mathcal{I}$ be $r+1$ independent vector fields. Their restrictions to $U$ are Killing fields tangent to $\mathscr{F}_{U}$, because $\mathscr{I}_{q}=\left(\mathscr{I}_{U}\right)_{q}$. Let $L$ be (an $m$-dimensional) leaf of $\mathscr{I}_{U}$. Thus there are constants $c_{1}, \cdots, c_{r+1}$ such that $\sum c_{j}\left(Y_{j}\right)_{1 L}=0$. Let us assume, for example, $c_{r+1} \neq 0$ and let $Y=: c_{r+1} Y_{r+1}-\sum_{j=1}^{r} c_{j} Y_{j}$. By Lemma 1, $Y_{\mid U}=0$. But $U$ is open on $M$; then $Y \equiv 0$, which contradicts the assumption on $Y_{1}, \cdots, Y_{r+1}$

Proposition 2. Let $(M, \mathscr{I})$ be a complete manifold with a codimension-one, $n$-dimensional, totally geodesic (not bundle-like) foliation. Then, dimension $G^{t} \leqq$ $(1 / 2) n(n-1)$.

Proof. It is enough to show the theorem for the universal cover of $(M, \mathscr{F})$. Therefore we may assume $M=L \times \boldsymbol{R}$ and $d s_{M}^{2}=d s_{L}^{2}+f^{2} d t^{2}$. Let $Y \in \mathcal{G}^{t}$. Thus, by Proposition $1,[Y, \partial t]=0$ and $\mathcal{G}^{t}$ has constant dimension along any $\mathscr{I}^{\perp}$-leaf. Therefore we may define $W=\left\{p \in L \mid\right.$ dimension $\mathcal{G}_{p x t}^{t}=n=$ dimension $\left.L\right\}$. If $W$ were dense in $L$, the foliation should be bundle-like, by Proposition 1 . Thus there is an open subset $U \subset L \backslash W$. If $\mathcal{G}_{U}:=\left\{X_{1 U}, \forall X \in \mathcal{G}^{t}\right\}$, then $\mathcal{G}_{U} \subset i(U)$ and $\forall p \in U$, dimension $\left(\mathscr{G}_{U}\right)_{p}=$ dimension $\mathcal{G}_{p}^{t} \leqq n-1$. By Lemma 2 , dimension $\mathcal{G}_{U} \leqq 1 / 2(n-1) n$. $\mathcal{G}^{t} \subset i(L)$ and $U$ is open on $L$, thus, independent vector fields on $\mathcal{G}^{t}$ give independent vector fields on $\mathcal{G}_{U}$ and dimension $\mathcal{G}^{t} \leqq$ dimension $\mathcal{G}_{U} \leqq(1 / 2) n(n-1)$.

Let us introduce some definitions :

Let $\mathcal{G}^{n}=:\left\{X \in T^{\perp}(\mathscr{I}) \cap \mathcal{G}\right\}$.

Let $\mathcal{G}=:\left\{Y \in T(\mathscr{I}) \mid \exists X \in \mathcal{G}\right.$ and $\left.Y=X^{t}\right\}$.

$\mathcal{G}^{n}$ and $\mathcal{G}$ are subalgebras of $\mathcal{G}$ and $\mathcal{G}^{t} \subset \mathcal{G}$. Moreover

Proposition 3. Dimension $\mathcal{G}^{n} \leqq 1$ and dimension $\mathcal{G}^{n}=1$ if and only if the universal cover $(\tilde{M}, \tilde{\mathcal{I}})$ is a warped product (in the sense that $\partial_{t} f=0$, in Theorem 1 ).

Proof. Let us assume $M$ to be simply connected. If $(M, \mathscr{F})$ is a warped product, it is clear from Proposition 1 that $\partial t$ is a Killing field.

Suppose now that $X=\lambda \partial t$ is a Killing field. Then $\partial_{t}(\lambda f)=0$ and $\lambda=\lambda(t)$. (Moreover, $\lambda$ never vanishes. See [4] for instance). If we reparametrize $\boldsymbol{R}$ 
with $\bar{t}=\int(1 / \lambda) d t$, then the metric reads as $d s^{2}=d s_{L}^{2}+(\lambda f)^{2} d \bar{t}^{2}$, which is a warped product and now $X=\partial \bar{t}$. Finally let $\mu \partial \bar{t}$ be another element of $\mathcal{G}$. Then, from ii) of Proposition 1 we may see that $\mu=$ cotstant.

\section{§ 3. Warped product foliations.}

We shall restrict now our attention to totally geodesic foliations with a warped product structure in the universal cover, (but not bundle-like). I.e., $\partial_{t} f=0$ in Theorem 1 , but $f \neq$ constant. This is equivalent to the fact that the 1-form $\theta$ associated to the vector field $\nabla_{N} N$ will be closed $\left(\theta(X)=:\left\langle\nabla_{N} N, X\right\rangle\right.$, for any vector field $X$ ):

Proposition 4. Let $(M, \mathscr{I})$ be a complete manifold with a codimension-one, totally geodesic foliation. Let $\tilde{M}$ be the universal cover of $M$. Then, the structure of $(\tilde{M}, \tilde{\mathscr{F}})$ stated in Theorem 1 is a warped product if and only if $d \theta=0$.

Proof. Suppose that $(\tilde{M}, \tilde{\mathscr{I}})$ is a warped product. We may consider in $M$ an orthonormal (local) basis $\left\{X_{1}, \cdots, X_{n}, N\right\}$ for $T(M)$, with $X_{i} \in T(\mathscr{I}) ;\left[X_{\imath}, \partial t\right]$ $=0, N=(1 / f) \partial t$; and such that $\partial_{t} f=0$. Then $\nabla_{N} N=-\Sigma\left(X_{i} \cdot \log f\right) X_{\imath}$ and $\theta\left(X_{\imath}\right)$ $=-X_{i} \cdot \log f$. Thus

$$
\begin{aligned}
& d \theta\left(X_{\imath}, X_{\jmath}\right)=-X_{\imath} X_{\jmath} \log f+X_{\jmath} X_{\imath} \log f+<\sum_{k=1}^{n}\left(X_{k} \cdot \log f\right) X_{k},\left[X_{\imath} X_{\jmath}\right]>=0, \\
& d \theta\left(X_{\imath}, \partial t\right)=\partial_{t} X_{\imath} \log f=X_{i} \partial_{t} \log f=0 .
\end{aligned}
$$

For the converse, let us assume $M$ to be simply connected. With the same notations as above, we have $X_{i} \partial t \log f=0, \forall i$. Then $f$ should be of the form $f=$ $e^{g} \cdot e^{h}$, where $g=g(t)$ and $h$ is defined on $L$, the generic leaf. With the change of parameter $\bar{t}=\int e^{g(t)} d t$ we obtain a warped product metric for $M$.

Remark. After the change of parameter, the manifold should remain of the form $M=L \times \boldsymbol{R}$. For if $M$ were equal to $L \times(a, b)$ and $a>-\infty$, for instance, we will consider geodesics with initial tangent vector $-\partial t$. Then, since leaves are totally geodesic submanifolds and translations in the direction of the 0t-axis are isometries (where they are defined), these geodesics should cross the extreme leaf $\{t=a\}$, which will contradicts the fact that $M$ is complete.

Proposition 5. Let $(M, \mathscr{F})$ be a complete manifold with a codimension-one, totally geodesic foliation, whose universal cover has a warped product structure. Let $X \in \mathcal{G}$. Then $\nabla_{N} X^{t}=k N$, with $k=$ constant.

Proof. Let us work in the universal cover of $(M, \mathscr{I})$. As $X=\left(X^{t}+\lambda \partial t\right) \in \mathcal{G}$, 


$$
\nabla_{N} X^{t}=-\left[X^{t}, N\right]=-\left[X^{t}, \frac{1}{f} \partial t\right]=\frac{X^{t} \cdot f}{f^{2}} \partial t=-\frac{\partial_{t}(\lambda f)}{f^{2}} \partial t=-\frac{\lambda^{\prime}}{f} \partial t .
$$

Actually, $\partial t$ is a Killing field. Thus, $\lambda^{\prime} \partial t=[\partial t, X] \in \mathcal{G}$ and $0=\partial_{t}\left(\lambda^{\prime} f\right)=\lambda^{\prime \prime} f$, so $\lambda^{\prime \prime}=0$. If we put $\lambda^{\prime}=-k$, then $\nabla_{N} X^{t}=(k / f) \partial t=k N$.

Remarks. (1) Observe that the constant $k$ verifies: $k=\left(X^{t} \log f\right)$. As a consequence, Killing fields tangent to the foliation are just vector fields $Y \in T(\mathscr{F})$ such that are Killing fields with respect to the metric of the leaves and verify $\nabla_{N} Y=0$. Moreover, every $X \in \mathcal{G}$ is of the form $X=X^{t}+(h-k t) f N$, for some constant $h$.

(2) The converse result is true when the manifold is simply connected (see $[8])$.

PROPOSITION 6. Let $(M . \mathscr{F})$ be a complete manifold with a codimension-one, totally geodesic foliation, whose universal cover is a warped product. Then dimension $\mathcal{G} \leqq$ dimension $\mathcal{G}^{t}+1$ and dimension $\mathcal{G}=$ dimension $\mathcal{I}+$ dimension $\mathcal{G}^{n}$.

Proof. If there is some $Y_{1} \in \mathcal{g} \backslash \mathcal{G}^{t}$, we may take $Y_{2}, \cdots, Y_{r}$ in order to form a basis $\left(Y_{1}, Y_{2}, \cdots, Y_{r}\right)$ of $g$. Thus, $\nabla_{N} Y_{2}=k_{i} N, \forall i$; and $k_{1} \neq 0$. Let $Z_{\jmath}:=$ $\left(\left(k_{j} / k_{1}\right) Y_{1}-Y_{j}\right), j: 2, \cdots, r$. It is easy to see that $\left(Y_{1}, Z_{2}, \cdots, Z_{r}\right)$ is a new basis of $g$ with $Z_{2}, \cdots, Z_{r} \in \mathcal{Q}^{t}$ and $X_{1}=Y_{1}+\left(h_{1}-k_{1} t\right) f N \in \mathcal{G}$.

For the second part, if $\mathscr{G}=\mathcal{G}^{t}$, then $\mathcal{G}=\mathfrak{g} \oplus \mathcal{G}^{n}$ and the result is obvious. Otherwise, let $X \in \mathcal{G} \backslash \mathcal{G}^{n}, X=X^{t}+(b-k t) f N$, where $\nabla_{N} X^{t}=k N$. But $X^{t} \in \mathcal{G}$, thus $X^{t}=a_{1} Y_{1}+\sum_{j=2}^{r} a_{j} Z_{\jmath}, \nabla_{N} X^{t}=a_{1} k_{1} N$ and $k=a_{1} k_{1}$. We have $X=a_{1} Y_{1}+\sum_{j=2}^{r} a_{j} Z_{j}+$ $\left(b-a_{1} k_{1} t\right) f N=a_{1} X_{1}+\sum_{j=2}^{r} a_{j} Z_{j}+\left(b-h_{1}\right) f N$. Then $X_{1}, Z_{2}, \cdots, Z_{r}$ and $\partial t=f N$ (if $\partial t$ is a global field) gives a basis of $\mathcal{G}$.

From Propositions 2, 3, 6, we can give now a complete description of the Lie algebra $\mathcal{G}$ :

THEOREM 2. Let $(M, \mathscr{I})$ be a complete manifold with a codimension-one, totally geodesic (not bundle-like) foliation of dimension $n$. Let $(\tilde{M}, \tilde{\mathscr{T}})$ denote the universal cover of $(M, \mathscr{I})$. If $(\tilde{M}, \tilde{\mathcal{F}})$ has a warped product structure, then dimension $\mathcal{G} \leqq 2+(1 / 2) n(n-1)$ and:

(1) For $(\tilde{M}, \tilde{\mathscr{T}})$ there are the following possibilities:

\begin{tabular}{|c|c|c|c|c|}
\hline$C A S E$ & $\operatorname{dim} \tilde{\mathcal{G}}^{n}$ & $\operatorname{dim} \tilde{\mathcal{G}}^{t}$ & $\operatorname{dim} \tilde{\mathfrak{I}}$ & $\operatorname{dim} \tilde{\mathcal{G}}$ \\
\hline$A$ & 1 & $m$ & $m+1$ & $m+2$ \\
\hline$B$ & 1 & $m$ & $m$ & $m+1$ \\
\hline
\end{tabular}

$$
\left(\text { with } 0 \leqq m \leqq \frac{1}{2} n(n-)\right)
$$


(2) and for $(M, \mathscr{I})$ :

\begin{tabular}{|c|c|c|c|c|c|}
\hline$C A S E$ & $C A S E$ on $\tilde{M}$ & $\operatorname{dim} \mathcal{G}^{n}$ & $\operatorname{dim} \mathcal{G}^{t}$ & $\operatorname{dim} \mathcal{G}$ & $\operatorname{dim} \mathcal{G}$ \\
\hline$A_{1}$ & $A$ & 1 & $m^{\prime}$ & $m^{\prime}+1$ & $m^{\prime}+2$ \\
\hline$A_{2}$ & $A$ & 1 & $m^{\prime}$ & $m^{\prime}$ & $m^{\prime}+1$ \\
\hline$A_{3}$ & $A$ & 0 & $m^{\prime}$ & $m^{\prime}+1$ & $m^{\prime}+1$ \\
\hline$A_{4}$ & $A$ & 0 & $m^{\prime}$ & $m^{\prime}$ & $m^{\prime}$ \\
\hline$B_{1}$ & $B$ & 1 & $m^{\prime}$ & $m^{\prime}$ & $m^{\prime}+1$ \\
\hline$B_{2}$ & $B$ & 0 & $m^{\prime}$ & $m^{\prime}$ & $m^{\prime}$ \\
\hline
\end{tabular}

$$
\left(\text { with } 0 \leqq m^{\prime} \leqq m \leqq \frac{1}{2} n(n-1)\right)
$$

We will give now some examples of all cases enumerated in Theorem 2 .

(A) Let $M_{1}=M^{\prime} \times \boldsymbol{R} \times \boldsymbol{R}, \quad \mathscr{T} \leftrightarrow\left(M^{\prime} \times \boldsymbol{R}\right) \times\{$ point $\}, \quad d s^{2}=d s_{M^{\prime}}^{2}+d x^{2}+e^{2 x} d t^{2}$. Here, $\partial t$ generates $\mathcal{G}^{n}$, whereas $\mathcal{G}^{t}=i\left(M^{\prime}\right)$ and $\partial x-t \partial t$ is a preserving Killing field neither tangent nor orthogonal to $\mathscr{F}$. Since $\boldsymbol{R}^{2}$ with the metric $d s^{2}=d x^{2}+e^{2 x} d t^{2}$ is isometric to the hyperbollic plane, $M_{1}$ is complete when $M^{\prime}$ is complete.

(B) Let $M_{2}=M^{\prime} \times \boldsymbol{R} \times \boldsymbol{R}, \mathscr{F} \leftrightarrow\left(M^{\prime} \times \boldsymbol{R}\right) \times\{$ point $\}, d s^{2}=d s_{M^{\prime}}^{2}+d x^{2}+e^{2(x+\sin x)} d t^{2}$. Also in this case, $\partial t$ generates $\mathcal{G}^{n}$ and $\mathcal{G}^{t}=i\left(M^{\prime}\right)$; but now $g=\mathcal{G}^{t}$. It is possible to see that $\boldsymbol{R}^{2}$ with this metric is a complete manifold, by solving differential equations of geodesics and aplying Theorem of Peano to extend these geodesics for any value of the parameter. Thus, $M_{2}$ will be complete when $M^{\prime}$ were a complete manifold.

$\left(A_{1}\right) \quad$ Let $M=M_{1} /\left\{\phi_{1}\right\}$ where $\phi_{1}(p, x, t)=(\tilde{\phi}(p), x, t)$ and $\tilde{\phi}$ is an isometry of $M^{\prime}$. Let us consider in $M$ metric and foliation induced by those in $M_{1}$.

$\left(A_{2}\right) \quad$ Let $M=M_{1} /\left\{\phi_{2}\right\}$, where now $\phi_{2}(p, x, t)=(p, x, t+1)$.

$\left(A_{3}\right) \quad$ Let $M=M_{1} /\left\{\phi_{3}\right\}$, where $\phi_{3}(p, x, t)=\left(p, x+1, e^{-1} t\right)$.

$\left(A_{4}\right) \quad$ Let $M=M^{\prime} \times T_{A}^{3}$, where $M^{\prime}$ is a complete manifold and $T_{A}^{3}$ is the so called "hyperbollic torus" and consider the product foliation $M^{\prime} \times \mathscr{I}^{\prime}, \mathscr{F}^{\prime}$ the usual codimension-one folation of $T_{A}^{3}$ (see for instance [6]). Here, $\mathcal{G}^{t}=i\left(M^{\prime}\right)$ and there are no Killing fields in the hyperbollic torus preserving $\mathscr{I}^{\prime}$.

Remark. This case $A_{4}$ may not occur in surfaces (see [8]).

$\left(B_{1}\right) \quad$ Let $M=M_{2} /\left\{\phi_{1}\right\}$, where $\phi_{1}(p, x, t)=(p, x, t+1)$.

$\left(B_{2}\right) \quad$ Let $M=M_{2} /\left\{\phi_{2}\right\}$, where now $\psi_{2}(p, x, t)=\left(p, x+2 \pi, t e^{-2 \pi}\right)$. 


\section{§4. Bundle-like foliations}

We shall consider now the special case when $Y f=0, \forall Y \in T(\mathscr{I})$. With a change of the parameter $t$, we may assume $f$ to be constant and consequently $\tilde{M}$ to be a riemannian product. Then it is easyly seen that $\tilde{\mathscr{I}}$ (and $\mathscr{I}$ also) is a bundle-like (totally geodesic) foliation. Conversely, when $\mathscr{F}$ is totally geodesic and bundle-like, the transverse one-dimensional foliation $\mathscr{F}^{\perp}$ is also totally geodesic and bundle-like (see [5]). It follows from Theorem A of [1] that the universal cover of $M$ is a riemannian product:

Proposition 7. Let $(M, \mathscr{F})$ be a complete manifold with a codimension-one, totally geodesic foliation. Then $\mathscr{I}$ is a bundle-like foliation if and only if the universal cover $(\tilde{M}, \tilde{\mathscr{F}})$ of $(M, \mathscr{F})$ is a riemannian product $\widetilde{L} \times \boldsymbol{R}$, foliated with leaves of the form $\widetilde{L} \times\{$ point $\}$.

Let us consider the Lie algebra $\mathcal{G}$ of Killing fields preserving the foliation. We will see that dimension $\mathcal{G} \leqq 1+(1 / 2) n(n+1)$, where $n=$ dimension $\mathscr{F}$.

PROPOSITION 8. Let $(M, \mathscr{I})$ be a complete manifold with a codimension-one, totally geodesic, bundle-like foliation. Then

i) $\lambda \partial t \in \mathcal{G}^{n} \Leftrightarrow \lambda \partial t=h N, h$ a constant.

ii) $\mathcal{G}=\mathcal{G}^{t} \oplus \mathcal{G}^{n}$.

Proof. i) Suppose that $\lambda \partial t \in \mathcal{G}^{n}$. Thus, by Propositions 1 and 7 it follows that $\lambda f=h$ constant and $\lambda \partial t=\lambda f N=h N$. The converse follows by the same argument.

ii) Let $X=X^{t}+\lambda \partial t=X^{t}+\lambda f N \in \mathcal{G}$. Then, by Propositions 1 and $7, \lambda f$ is constant and $\lambda \partial t \in \mathcal{G}^{n}$. So $X^{t}=X-\lambda f N \in \mathcal{G} \cap T(\mathscr{F})=\mathcal{G}^{t}$.

As an obvious consequence, we have:

COROLLARY 1. Let $(M, \mathscr{F})$ be a complete manifold with a codimension-one, totally geodesic, bundle-like foliation of dimension $n$. Then $1 \leqq$ dimension $\mathcal{G} \leqq 1+$ $(1 / 2) n(n+1)$.

Remark. In fact, the second inequality in Corollary 1 holds for any codimension-one, bundle-like foliation, also in the non-totally geodesic case (see [8]).

Let us give some examples which prove that inequalities in Corollary 1 are as fine as possible:

The $(n+1)$-dimensional euclidean space, foliated by parallel hyperplanes, gives us an example with dimension $\mathcal{G}=1+(1 / 2) n(n+1)$. Here $\mathcal{G}$ is generated by $\left\{i\left(\boldsymbol{R}^{n}\right) \cup\left\{\partial x_{n+1}\right\}\right\}$.

Now let $G$ be the group generated by the isometries of the euclidean 3-space $\phi$ and $\phi$, where $\phi(x, y, t)=(-x,-y, t+1)$; and $\phi(x, y, t)=(x+1, y, t)$. Let $M=$ 
$\left(\boldsymbol{R}^{2} \times \boldsymbol{R}\right) / G$, with the foliation induced by the one in $\boldsymbol{R}^{3}$ whose leaves are of the form $\boldsymbol{R}^{2} \times\{$ point $\}$. Then, $\mathcal{G}$ is generated by $\left\{\pi_{*}(\partial t)\right\}$ and has dimension one.

\section{REFERENCES}

[1] R. A. Blumenthal And J. J. Hebda, De Rham decomposition theorems for foliated manifolds, Ann. Inst. Fourier, Grenoble, 33 (1983), 183-198.

[2] R. A. Blumenthal and J.J. Hebda, Ehresmann Connections for Foliations, Indiana Math. J. 33 (1984), 597-611.

[3] C. Currás-Bosch, Killing vector fields and holonomy algebras, Proc. Amer. Math. Soc., 90 (1984), 97-102.

[4] C. Currás-Bosch, The geometry of totally geodesic foliations admitting Killing field, Tôhoku Math. J. 40 (1988), 535-548.

[5] D. L. Johnson AND L.B. Whitt, Totally Geodesic Foliations, J. Differential Geom. 15 (1980), 225-235.

[6] J. MEYER, e-foliations of codimension two, J. Differential Geom. 12 (1977), 583-594.

[7] G. Oshikiri, Totally geodesic foliations and Killing fields, Tôhoku Math. Journ, 35 (1983), 387-392.

[8] A. RAS, Foliacions totalment geodèsiques de codimensió 1 i camps de Killing (Thesis). Universitat de Barcelona, 1988.

[9] B. L. Reinhart, Foliated manifolds with bundle-like metrics, Ann. of Math. (2) 69 (1959), 119-132.

ANTONI RAS-SABIDÓ

Departament de Matemàtica Aplicada i Telemãtica.

Universitat Politecnica de Catalunya.

Eduard Maristany, s. n. ; 08800-Vilanova i la Geltrú, Spain 